\title{
Increased Incidence of Hypothyroidism in Primary Biliary Cirrhosis
}

\author{
GRACE H. ELTA, MD, ROBERT A. SEPERSKY, MD, MICHAEL J. GOLDBERG, MD, \\ CHRISTINE M. CONNORS, KENNETH B. MILLER, MD, and MARSHALL M. KAPLAN, MD
}

\begin{abstract}
We examined the thyroid status of 58 patients with primary biliary cirrhosis ( $P B C)$ using total serum thyroxin, thyroid hormone binding ratio, free thyroxin index, serum TSH, antithyroglobulin, and antimicrosomal antibodies. Seven patients were known to be hypothyroid prior to the diagnosis of PBC. Six additional patients were found to have biochemical evidence of hypothyroidism. The prevalence of hypothyroidism was $12 \%$ if we include only those six PBC patients with newly diagnosed hypothyroidism or $22 \%$ if we include all 13 patients. Five of the 58 patients had evidence for an elevation of thyroid hormone binding capacity. Three hypothyroid patients had normal total thyroxins with low thyroid hormone binding ratios. Two euthyroid patients had elevated total $T_{4} S$ with low thyroid hormone binding ratio and normal FTI. The prevalence of positive antimicrosomal antibodies was $34 \%$, including 11 euthyroid PBC patients. The prevalence of positive antithyroglobulin antibodies was $20 \%$ including five euthyroid patients. There was no association between HLA DR3 or DR5 and the patients with hypothyroidism and or antithyroid antibodies. Because fatigue, lethargy, and anorexia as well as hypercholesterolemia are common features of both hypothyroidism and $P B C$, patients with $P B C$ should be screened for evidence of thyroid dysfunction. Thyroid disease may precede the diagnosis of PBC by several years. Therefore, the development of cholestatic liver disease in a patient with known autoimmune thyroiditis should arouse suspicion of $P B C$.
\end{abstract}

Primary biliary cirrhosis ( $\mathrm{PBC}$ ) is a progressive liver disease of unknown etiology for which only symptomatic treatment exists (1). As in most chron-

Manuscript received November 20, 1982; revised manuscript received April 6, 1983; accepted May 2, 1983.

From the Gastroenterology Division, Department of Medicine, Tufts-New England Medical Center, Boston, Massachusetts 02111; and Tissue Typing Laboratory, Brigham and Women's Hospital, Boston, Massachusetts 02115.

Grace Elta is currently at the Gastroenterology Division, Department of Medicine, University of Michigan, Ann Arbor, Michigan 48109.

Michael Goldberg is currently at the Louis A. Weiss Memorial Hospital and Department of Medicine, University of Illinois School of Medicine, Chicago, Illinois.

This work was supported by Training Grant AMO7024 and Clinical Study Unit Grant RR 300054 from the National Institutes of Health.

Address for reprint requests: Dr. Marshall M. Kaplan, TuftsNew England Medical Center, 171 Harrison Avenue, Box 233, Boston, Massachusetts 02111. ic illnesses, detection and treatment of any associated diseases are very important in the overall care of patients with PBC. Among the array of symptoms manifested by patients with this disease are fatigue, lethargy, and anorexia (2). These are nonspecific symptoms associated with a large number of diseases. In patients with $\mathrm{PBC}$ these symptoms are usually attributed to the chronic liver disease.

Patients with PBC have an increased incidence of several autoantibodies including antismooth muscle (4), antinuclear $(4,5)$, antimitochondrial (3-5), and antithyroglobulin (5) antibodies. In addition, autoimmune disorders such as Sjogren's syndrome, rheumatoid arthritis, CRST syndrome, and autoimmune thyroiditis occur with increased frequency in patients with $\mathrm{PBC}(6)$. Hypothyroidism has been noted to occur in PBC (7). Recently, biochemical evidence of hypothyroidism was found in $18 \%$ of a 
ELTA ET AL

Table 1. Primary Biliary Cirrhosis Patients with Presumptive or Definite Thyroid Disease*

\begin{tabular}{|c|c|c|c|c|c|c|c|}
\hline Patient & $\begin{array}{l}P B C \\
\text { stage }\end{array}$ & $\begin{array}{l}A M c A \\
\text { titer }\end{array}$ & $\begin{array}{c}\text { ATG } \\
\text { titer }\end{array}$ & $\begin{array}{c}T S H \\
(N I<8.0 \\
\mu U / m l)\end{array}$ & $\begin{array}{c}\text { Total } T_{4} \\
(\mathrm{Nl}: 4.2-12 \\
\mu \mathrm{g} / 100 \mathrm{ml})\end{array}$ & $\begin{array}{c}T H B R \\
(N 1: 0.82-1.2)\end{array}$ & $\begin{array}{c}F T I \\
(N 1: 5.5-11.5)\end{array}$ \\
\hline \multicolumn{8}{|c|}{ Newly diagnosed hypothyroidism } \\
\hline KS & III & $1: 400$ & $1: 100$ & 90.5 & 0.2 & 0.60 & 1.2 \\
\hline $\mathrm{MG}$ & III & $1: 1600$ & $1: 6400$ & $14: 3$ & 6.3 & 0.77 & 4.9 \\
\hline $\mathrm{BL}$ & II & $1: 6400$ & $1: 100$ & $15: 1$ & 3.3 & 0.9 & 3.0 \\
\hline JT & II & $1: 25,600$ & $1: 100$ & 22.4 & 6.0 & 0.8 & 4.8 \\
\hline HB & IV & $1: 1600$ & $1: 100$ & 12.3 & 4.5 & 1.02 & 4.6 \\
\hline SK & I & $1: 102,400$ & neg & 11.3 & 4.0 & 0.94 & 3.8 \\
\hline \multicolumn{8}{|c|}{ Taking thyroid replacement medicine } \\
\hline $\mathrm{MC}$ & IV & neg & neg & & & & \\
\hline MR & III & $1: 1600$ & $1: 6400$ & & & & \\
\hline $\mathrm{AM}$ & III & neg & neg & & & & \\
\hline ER & III & $1: 25,600$ & neg & & & & \\
\hline $\mathrm{MD}$ & IV & $1: 1600$ & $1: 400$ & & & & \\
\hline RK & II & neg & neg & & & & \\
\hline RK & IV & neg & neg & & & & \\
\hline \multicolumn{8}{|c|}{ Euthyroid AMcA and/or ATG positive } \\
\hline $\mathrm{MT}$ & II & $1: 25,600$ & $1: 6400$ & 2.5 & 9.6 & 0.9 & 9.6 \\
\hline PW & I & $1: 25,600$ & $1: 400$ & 7.4 & 7.9 & 0.99 & 7.8 \\
\hline LM & III & $1: 400$ & $1: 400$ & 5.3 & 5.8 & 0.88 & 5.1 \\
\hline JC & I & $1: 100$ & neg & 2.5 & 8.3 & 1.09 & 9.1 \\
\hline PM & III & $1: 400$ & neg & 5.9 & 8.0 & 0.88 & 6.8 \\
\hline $\mathrm{CM}$ & IV & $1: 400$ & neg & 3.3 & 9.0 & 0.95 & 8.6 \\
\hline JN & III & $1: 100$ & neg & 5.7 & 7.3 & 0.99 & 7.2 \\
\hline $\mathrm{JC}$ & III & $1: 6400$ & neg & 2.4 & 12.5 & 0.98 & 12.3 \\
\hline FE & II & $1: 400$ & neg & 2.5 & 6.5 & 1.04 & 6.8 \\
\hline AL & IV & $1: 400$ & neg & 7.1 & 11.0 & 1.04 & 11.3 \\
\hline $\mathrm{AD}$ & IV & neg & 1:100 & 5.2 & 6.3 & 0.83 & 5.2 \\
\hline YK & I & $1: 1600$ & $1: 400$ & 3.1 & 7.7 & 1.5 & 8.9 \\
\hline
\end{tabular}

${ }^{*} \mathrm{AMcA}=$ antimicrosomal antibody $; \mathrm{ATG}=$ antithyroglobulin antibody; $\overline{\mathrm{TSH}}=$ thyroid stimulating hormone; $\mathrm{THBR}=$ thyroid hormone binding ratio; FTE $=$ free thyroxin index (total $\mathrm{T}_{4} \times \mathrm{THBR}$ ); total $\mathrm{T}_{4}=$ total serum thyroxin; neg = negative.

group of 95 patients with PBC (8). There have been conflicting reports regarding thyroid hormone binding capacity in PBC $(7,8)$. To explore further and confirm these findings, we screened our PBC patients with total serum thyroxin $\left(\mathrm{T}_{4}\right)$, thyroid hormone binding ratio (THBR), free thyroxin index (FTI), thyroid stimulating hormone (TSH), antithyroglobulin antibody (ATA), and antimicrosomal antibody (AMcA).

\section{MATERIALS AND METHODS}

All patients followed at New England Medical Center between January 1978 and June 30, 1982, with well characterized primary biliary cirrhosis were included in this study. All had liver biopsies consistent with PBC and common bile duct patency demonstrated by either intravenous cholangiography, endoscopic retrograde cholangiography, or laparotomy. Histologic staging of the disease was done by currently accepted criteria (9). The group consisted of 53 females and five males. Antimitochondrial antibody was positive in 53 patients (91\%). Seven of the 58 patients were known to be hypothyroid prior to the diagnosis of their liver disease. All seven were taking thyroid hormone replacement.

Total serum thyroxin $\left(\mathrm{T}_{4}\right)$ was measured by a standard radioimmunoassay. Normal ranges are listed in Table 1 and are \pm 2 SD of a previously obtained normal distribution. Thyroid hormone binding ratio was measured as a triiodothyronine uptake $\left(\mathrm{T}_{3} \mathrm{U}\right)$ procedure which uses talc absorption of free $\left[{ }^{125} \mathrm{I}\right] \mathrm{T}_{3}$ after adding the serum sample to a known amount of $\left.{ }^{125} \mathrm{I}\right] \mathrm{T}_{3}$. The remaining $\left[{ }^{125} \mathrm{I}\right] \mathrm{T}_{3}$ is then quantified. The thyroid binding ratio is expressed as a ratio of counts of $\left[{ }^{125} \mathrm{I}\right] \mathrm{T}_{3}$ of pooled normal serum to the counts of $\left.{ }^{125} \mathrm{I}\right] \mathrm{T}_{3}$ of patient serum. A pool of euthyroid sera is collected over several weeks by freezing the individual sera and then collectively mixing and refreezing the pool in small aliquots of $0.5 \mathrm{ml}$. The free thyroxin index is determined by multiplying the thyroid hormone binding ratio times the total serum thyroxin. Antithyroglobulin antibody and antimicrosomal antibody were measured by hemagglutination using the Sera-Tek antibody kits (Ames, Elkhart, Indiana). Thyroid stimulating hormone (TSH) was measured with a human TSH RIA kit (Abbott Laboratories, North Chicago, Illinois).

HLA-DR typing was done as follows: B and T lymphocytes were isolated from peripheral blood using a nylonwool column technique from a Ficoll-Hypaque prepared total lymphocyte fraction (10). Lymphocyte assays with 
peripheral T cell lymphocytes were performed using the standard NIH technique (11). Lymphocyte assays with peripheral B cells were performed according to the standard NIH technique with increased incubation times of 1 $\mathrm{hr}$ with sera and cells and $2 \mathrm{hr}$ with complement at room temperature. The $58 \mathrm{PBC}$ patients were compared with 63 members of a local control panel and with 3811 individuals studied in the 8th International Workshop (12).

\section{RESULTS}

None of our PBC patients had a palpable goiter at the time of this study, although one patient had had a goiter surgically resected years before her $\mathrm{PBC}$ was diagnosed. Seven of our 58 patients were known to be hypothyroid prior to the diagnosis of their liver disease. In all, the diagnosis was based on low serum levels of $\mathrm{T}_{4}$. More complete testing of thyroid function was not done. These patients were all taking thyroid hormone replacement. Thyroid function tests and serum TSH determinations revealed an additional six patients with biochemical hypothyroidism. One of these six patients had profound depression of her serum thyroxin and had a free thyroxin index of 1.2. The incidence of hypothyroidism in our PBC population was $22 \%$ if we include all 13 patients in the analysis. If we include only the six whose hypothyroidism was diagnosed after the onset of the PBC but whose diagnosis of thyroid dysfunction was more securely established, the incidence is $12 \%$. In either instance the incidence of hypothyroidism is much larger than the $1.4-1.9 \%$ expected in a comparable group of women (13) or the $5.9 \%$ found in a group of healthy men and women older than 60 years (14).

Of the 13 patients with hypothyroidism, 10 had positive antimicrosomal antibodies and seven had positive antithyroglobulin antibodies. One of the patients taking thyroid replacement therapy who was negative for antithyroglobulin and antimicrosomal antibodies had previously had a subtotal thyroidectomy for goiter with pathologic examination revealing autoimmune thyroiditis. In addition to the patients found to be hypothyroid, 11 patients were found to have positive antimicrosomal and five positive antithyroglobulin antibodies. Two of these had low FTIs, 5.1 and 5.2, respectively, suggesting borderline hypothyroidism. Three of the six newly diagnosed hypothyroid patients had total $\mathrm{T}_{4} \mathrm{~S}$ in the normal range. All, however, had low free thyroxin indexes. Two of the 45 euthyroid patients had elevated total serum $\mathrm{T}_{4} \mathrm{~s}$ with low thyroid hormone binding ratios and normal FTI and free serum thyroxins. Table 1 presents the clinical data, PBC histologic stage, and autoantibody status of the hypothyroid patients and the patients with positive autoantibodies. There was no correlation in our 58 patients between PBC stage and presence of hypothyroidism. There was no evidence for increased frequency of DR3 and DR5 in either the entire PBC group or in those with hypothyroidism and/or antithyroid antibodies when compared to the local or the international control gorups. Likewise there was no clustering of the hypothyroid PBC patients into any other HLA locus. An association between autoimmune thyroiditis and HLA-DR3 and DR5 has been previously noted $(15,16)$.

Because hypercholesterolemia is a feature of both thyroprivic hypothyroidism and PBC, we followed the serum cholesterol levels in three of the four newly diagnosed hypothyroid patients as they were placed on thyroid hormone replacement to see if the levels would decrease from pretreatment values. Two had stage III disease and one had stage II. As depicted in Figure 1, serum cholesterol values were observed to decrease in all three patients following the institution of thyroid hormone replacement. All three noted symptomatic improvement, primarily a lessening of fatigue. However, one patient also had a sustained improvement in liver function tests during the ensuing year with bilirubin falling from 8.2 to $3.5 \mathrm{mg} / \mathrm{dl}$, alkaline phosphatase from 1598 to $878 \mathrm{IU}$, aspartate aminotransferase from 218 to 150 and cholesterol from 690 $\mathrm{mg} / \mathrm{dl}$ to 215 .

\section{DISCUSSION}

Our data indicate that hypothyroidism is a frequent finding in PBC. Its onset may precede or follow that of $\mathrm{PBC}$. The diagnosis of hypothyroidism preceded the diagnosis of $\mathrm{PBC}$ by an interval ranging from 18 months to 9 years in seven patients and followed the diagnosis of PBC in six patients by 1-8 years. The incidence of hypothyroidism was $22 \%$, similar to the $18 \%$ incidence reported by Crowe et al (8). The six patients in whom hypothyroidism was newly detected each had prominent symptoms of fatigue, lethargy, and anorexia which had previously been ascribed to their liver disease. Upon treatment for their thyroid disease, these symptoms improved. We believe that these nonspecific symptoms were at least partially due to the underlying hypothyroidism. In addition, elevated serum cholesterol levels, also believed to be due to 


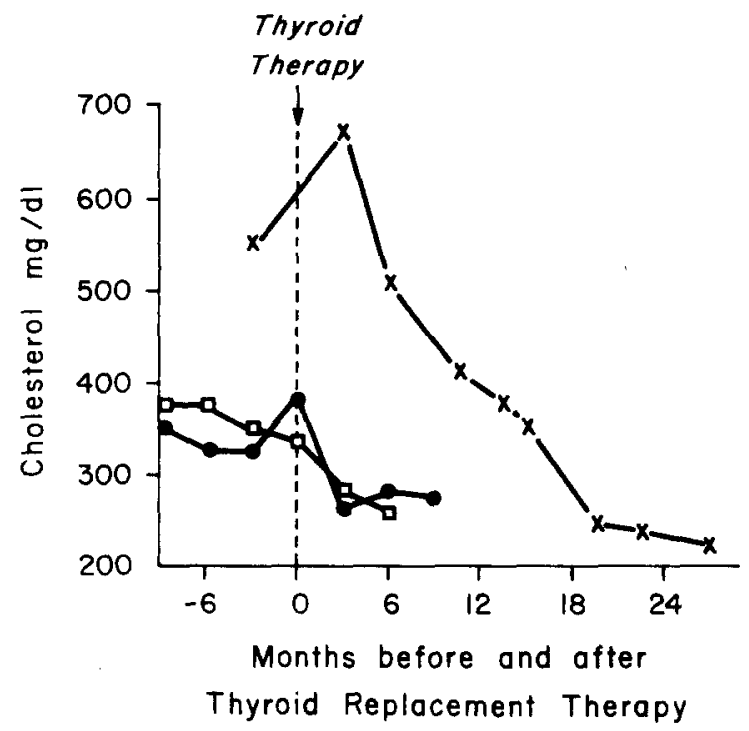

Fig 1. Response of serum cholesterol to thyroid replacement therapy in three patients with hypothyroidism and primary biliary cirrhosis.

PBC, decreased in the three hypothyroid patients in whom it was serially measured after the patients were started on thyroid replacement therapy. The distinct decrease in the degree of hypercholesterolemia which followed the institution of thyroid replacement therapy suggests that the hypothyroid state was at least partially responsible for the elevated serum cholesterol. It is unlikely that the decrease in serum cholesterol was due to an independent effect of thyroxin therapy since thyroxin must be given in pharmacologic doses to normal subjects to lower serum cholesterol (17).

We found a $34 \%$ prevalence of positive antimicrosomal antibodies and a $20 \%$ prevalence of antithyroglobulin antibodies, similar to the incidence reported in previous studies $(5,8)$. Not unexpectedly, nine of 13 of the hypothyroid patients in our series had positive antimicrosomal antibodies, and eight had positive antithyroglobulin antibodies. One of the patients who was negative for both antibodies had a goitrous gland removed 9 years prior to the diagnosis of $\mathrm{PBC}$ with pathologic examination consistent with autoimmune thyroiditis. It is known that autoimmune thyroiditis may occur without detectable serum thyroid autoantibodies (18). However, it is also known that antithyroid antibodies fall and may become negative with thyroid replacement therapy. Hence, four of the seven patients with preexisting hypothyroidism and no antithyroid anti- bodies may have had such antibodies at the time their thyroid disease was first diagnosed.

Schussler et al reported finding increased thyroid hormone binding proteins in PBC and chronic active hepatitis (7). They noted that this increase might result in a failure to recognize hypothyroidism or cause an overdiagnosis of hyperthyroidism on the basis of serum thyroxin alone. In our series, three of our six newly diagnosed hypothyroid patients had normal total $\mathrm{T}_{4}$ determinations accompanied by the low FTI. Not shown in Table 1, two euthyroid patients had elevated total $\mathrm{T}_{4} \mathrm{~s}$ with decreased thyroid hormone binding ratios. Therefore, five of our 58 patients $(9 \%)$ had evidence suggestive of increased thyroid binding. This finding is in agreement with Schussler et al (7) and is different from Crowe et al (8) who found no evidence of increased thyroid hormone binding in PBC.

Our results strongly support the concept that thyroid disease is frequently associated with PBC and may relate to an autoimmune process involving both the thyroid gland and the hepatic bile ducts. We detected 12 patients with positive antimicrosomal and/or antithyroglobulin antibodies who were euthyroid by standard tests. Two of these 12 had borderline low free thyroxin index determinations, but their serum TSH levels were not frankly elevated. It will be of interest to follow these patients with periodic TSH levels since the proportion of patients with autoimmune thyroiditis that become biochemically hypothyroid increases with time (19). Alternatively, TRH stimulation tests can be employed to detect marginal hypothyroidism by observing an exaggerated TSH response (20).

The symptoms of hypothyroidism can overlap with those of PBC. Hypercholesterolemia is a feature of both conditions. Clearly, all patients with PBC should be screened with standard thyroid function tests and have their antimicrosomal antibody status determined. This antibody is more sensitive than the antithyroglobulin antibody in screening for autoimmune thyroiditis (21). Euthyroid patients who have positive antithyroglobulin and antimicrosomal antibodies should be observed periodically for the onset of biochemical hypothyroidism, evidenced by either a rising TSH level or an exaggerated response to TRH stimulation. Conversely, since many of our patients had hypothyroidism detected years before the diagnosis of PBC, the discovery of an elevated alkaline phosphatase or the development of pruritus or jaundice in a patient with previously diagnosed autoimmune thy- 
roiditis or hypothyroidism should immediately suggest the possibility of PBC. Awareness of the association between these two diseases will allow earlier diagnosis.

\section{REFERENCES}

1. Matloff DS, Alpert E, Resnick RH, Kaplan MM: A prospective trial of D-penicillamine in primary biliary cirrhosis. $\mathrm{N}$ Engl J Med 306:319-326, 1982

2. Christensen E, Crowe J, Doniach D, Popper H, Ranek L, Rodes J, Tygstrup N, Williams R: Clinical pattern and course of disease in primary biliary cirrhosis based on an analysis of 236 patients. Gastroenterology 78:236-246, 1980

3. Tong MJ, Nies KM, Reynolds TB, Quismoro FP: Immunologic studies in familial primary biliary cirrhosis. Gastroenterology 71:305-307, 1976

4. Sherlock S: Primary biliary cirrhosis. Prog Liver Dis 5:559574, 1976

5. Feizi T, Naccarato R, Sherlock S, Doniach D: Mitochondrial and other tissue antibodies in relatives of patients with patient biliary cirrhosis. Clin and Exp Immunol 10:609-622, 1975

6. Clarke AK: Rheumatic disorders in primary biliary cirrhosis. Ann Rheum Dis 37:42-47, 1978

7. Schussler G, Schaffner F, Korn F: Increased serum thyroid hormone binding and decreased free hormone in chronic active hepatitis. N Engl J Med 299:510-515, 1978

8. Crowe JP, Christensen E, Butler J, Wheeler P, Doniach D, Keenan J, Williams R: Primary biliary cirrhosis: The prevalence of hypothyroidism and its relationship to thyroid autantibodies and sicca syndrome. Gastroenterology $78: 1437-1441,1980$
9. Scheuer PJ: Liver Biopsy Interpretation, 2nd ed, Baltimore, Williams \& Wilkins, 1973

10. Lowry RP, Person AE, Goguen JE, Carpenter CB, Garovoy MR: Technical modification for ADCC versus separated T and $B$ lymphocytes: $T$ and $B$ cell separations by nylon wool columns. Transplant Proc 10:833-837, 1978

11. DHEW Publication No. NIH 76-545, 1976, p 22

12. Terasaki PI: Histocompatibility testing 1980. Los Angeles, California. UCLA Tissue Typing Laboratory, Regents of the University of California, 1980, pp 962-963

13. Tunbridge WMG: Screening for thyroid disease in the community. Thyroid Today 5:1-4, 1982

14. Sawin CT, Chopra D, Azizi F, Mannix JE, Bacharach P: The aging thyroid: Increased prevalence of elevated serum thyroxin levels in the elderly. JAMA $242: 247-253,1979$

15. Moens H, Farid NR: Hashimoto's thyroiditis is associated with HLA-DRW3. N Engl J Med 299: 133-134, 1978

16. Weissel M, Höfer R, Zasmeta H, Mayr WR: HLA-DR and Hashimoto's thyroiditis. Tissue Antigens 16:256-257, 1980

17. Schneeberg NG, Herman E, Menduke H, Altshuler NK: Reduction of serum cholesterol by sodium dextrothyroxin in euthyroid subjects. Ann Intern Med 56:265-275, 1962

18. Roitt IM, Doniach D: Human autoimmune thyroiditis: Serological studies. Lancet 2:1027, 1958

19. Gordin A, Lamberg BA: Natural course of symptomless autoimmune thyroiditis. Lancet 2:1234, 1978

20. Ingbar SH, Woeber KA: The thyroid gland, Chap. 4. In Textbook of endrocrinology, 5th ed. RH Williams (ed). WB Saunders, 1974

21. Abreau CM, Vagenakis AG, Roti E, Braverman LE: Clinical evaluation of a hemagglutination method for microsomal and thyroglobulin antibodies in autoimmune thyroid disease. Ann Clin Lab Sci 7:73-38, 1977 